\title{
Absolute calibration of photon-number-resolving detectors with an analog output using twin beams
}

\author{
Jan Peřina, Jr., 1, (a) Ondřej Haderka, ${ }^{2}$ Alessia Allevi, ${ }^{3, b}$ and Maria Bondani, ${ }^{4}$, \\ ${ }^{1)}$ RCPTM, Joint Laboratory of Optics of Palacký University and Institute of Physics AS CR, 17. listopadu 12, \\ 77146 Olomouc, Czech Republic. \\ ${ }^{2)}$ Institute of Physics AS CR, Joint Laboratory of Optics of Palacký University and Institute of Physics AS CR, \\ 17. listopadu 12, 772 07 Olomouc, Czech Republic. \\ 3) Dipartimento di Scienza e Alta Tecnologia, Università degli Studi dell'Insubria, I-22100 Como, Italy. \\ ${ }^{4)}$ Istituto di Fotonica e Nanotecnologie, CNR-IFN, I-22100 Como, Italy.
}

\begin{abstract}
A method for absolute calibration of a photon-number resolving detector producing analog signals as the output is developed using a twin beam. The method gives both analog-to-digital conversion parameters and quantum detection efficiency for the photon fields. Characteristics of the used twin beam are also obtained. A simplified variant of the method applicable to fields with high signal to noise ratios and suitable for more intense twin beams is suggested.
\end{abstract}

PACS numbers: 03.65.Wj, 42.50.Ar, 42.65.Lm

Detection of photons by detectors sensitive to electricfield intensities represents the basic and by far the most important diagnostic tool for optical fields $\stackrel{1}{*}$. That is why quality and capabilities of optical detectors have continuously attracted a great deal of attention in the whole history of modern optics. Construction of detectors with single-photon resolution (photomultipliers, semiconductor single-photon counting modules) represented a milestone in this effort. The ability to build photonnumber resolving detectors (PNRD) reached some years ago was undoubtedly the next milestone. Due to advanced technologies available that time, several kinds of PNRDs have been designed. Some of them, including optical-fiber-loop detectors ${ }^{2-4}$ and intensified CCD cameras ${ }^{5}$, produce a digitized signal that directly determines the number of detected photons called photoelectrons. However, several kinds of detectors give only analog outputs and so this output has to be somehow mapped onto integer photo-electron numbers. Superconducting bolometers ${ }^{6.7}$, transition-edge sensors ${ }^{8}$, silicon photomultipliers $\underline{-12}$ and hybrid photo-detectors $(\mathrm{HPD})^{12,13}$ can be mentioned as typical examples. Calibration of such detectors inevitably requires at least two constants, one giving the probability of detecting a photon by creating a photo-electron [absolute quantum detection efficiency (QDE)] and one providing the mapping between the analog signal and photo-electron numbers. Reliable determination of these constants then opens the door for measuring quantum properties of optical fields at the level of individual photons 14 . As a result it gives qualitatively better tools for the experimental analysis of nonclassical fields.

There exist classical approaches for absolute detector calibration for both kinds of detectors. However, an elegant calibration method based on weak entangled two-

\footnotetext{
a) Electronic mail: jan.perina.jr@upol.cz

b) CNISM UdR Como, I-22100 Como, Italy.
}

photon fields $\frac{15}{5}$ and developed for single-photon resolving detectors over more than thirty years $\underline{16}$ has provided an inspiration also for PNRDs with digitized outputs. Contrary to weak two-photon fields, the method naturally uses more-intense twin beams (TB) with mean photonpair numbers larger than one $\frac{17,18}{}$. Here, we further generalize the method to account for analog outputs of the remaining kinds of PNRDs. Similarly to the approach presented in $\underline{18}$, the method also provides the characteristics of the used TB. We note that another generalization of the original method has been given mixing the analyzed field with a heralded single-photon field ${ }^{8}$.

We demonstrate the method by considering a suitable TB measured simultaneously by two HPDs 12,13 . HPDs provide resolution for small numbers of detected photoelectrons. Current coming from the semiconductor cathode of an HPD shows several peaks that can be assigned to different photo-electron numbers. After amplification and conversion of the measured real values of voltages into (arbitrarily scaled) dimensionless voltages, we obtain pairs $\left(v_{s}, v_{i}\right)$ of dimensionless voltages characterizing simultaneous detections in the signal and idler beams. By repeating the measurement $N$ times, we obtain the moments of the ensemble of real values of voltages $\left(v_{s, j}, v_{i, j}\right)$ :

$$
\left\langle v_{s}^{k} v_{i}^{l}\right\rangle_{a}=\frac{1}{N} \sum_{j=1}^{N} v_{s, j}^{k} v_{i, j}^{l}, \quad k, l=0,1, \ldots
$$

Symbol \langle\rangle$_{a}$ denotes a mean value taken over the values of measured quantities. As usual for reasonable numbers $N$ of measurement repetitions, only the considered first and second moments are reached with sufficient precision ${ }^{18,19}$.

Quantum nature of the detected light composed of photons, however, requires the introduction of discrete quantities instead of the real-valued voltages $v_{s, j}$ and $v_{i, j}$. That is why we have to assign a certain interval of voltages for each state with a fixed number of detected photo-electrons. To do this we define voltage windows of widths $\delta v_{s}$ and $\delta v_{i}$ for the signal and idler voltage axes, respectively, and assign discrete values $m_{c, j}$ to the mea- 


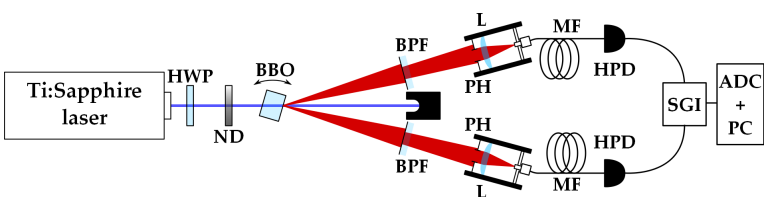

FIG. 1. Scheme of the experimental setup; HWP - half-wave plate, ND - neutral-density filter, $\mathrm{BBO}-\mathrm{BaB}_{2} \mathrm{O}_{4}$ nonlinear crystal, BPF - band-pass filter, $\mathrm{PH}$ - pinhole, L - lens, MF - multi-mode fiber, HPD - hybrid photo-detector, SGI - synchronous-gated integrator, ADC - analog-to-digital converter.

sured values $v_{c, j}(c=s, i)$ by the formula

$$
m_{c, j}=\bmod \left[v_{c, j} / \delta v_{c}+1 / 2\right] .
$$

Photo-electron histogram $f_{\delta v}\left(m_{s}, m_{i}\right)$ depending on windows' widths $\delta v_{s}$ and $\delta v_{i}$ can then be built from the ensemble of pairs $\left(m_{s, j}, m_{i, j}\right)$.

Using a classical approach, windows' widths $\delta v_{s}$ and $\delta v_{i}$ can be determined invoking a special calibration procedure $\frac{13,20}{2}$. The measurement of TBs, however, suggests an alternative way for their determination. As TBs contain predominantly photon pairs, values of voltages $v_{s, j}$ and $v_{i, j}$ are correlated despite final detectors' QDEs $\eta_{s}$ and $\eta_{i}$ in both detection arms. So also signal and idler photo-electron numbers $m_{s}$ and $m_{i}$ are correlated. This correlation is quantified by covariance $c_{m, \delta v}$, which depends on widths $\delta v_{s}$ and $\delta v_{i}$ :

$$
c_{m, \delta v}=\left\langle\Delta m_{s} \Delta m_{i}\right\rangle / \sqrt{\left\langle\left(\Delta m_{s}\right)^{2}\right\rangle\left\langle\left(\Delta m_{i}\right)^{2}\right\rangle} .
$$

where $\Delta m=m-\langle m\rangle$ and $\left\langle m_{s}^{k} m_{i}^{l}\right\rangle=\sum_{j=1}^{N} m_{s, j}^{k} m_{i, j}^{l} / N$, $k, l=0,1, \ldots$. Covariance $c_{m, \delta v}$ attains its maximum for certain values of windows' widths $\delta v_{s}$ and $\delta v_{i}$. These values are optimal as they maintain the pair-wise character of detected twin beams in the best possible way.

In the experiment shown in Fig. 1, TB was generated in a type $\mathrm{I} \mathrm{BaB}_{2} \mathrm{O}_{4}$ crystal (cut angle $\vartheta_{c}=48 \mathrm{deg}$ ) pumped by the third harmonics (at $266 \mathrm{~nm}$ ) of a cavity-dumped Ti:Sapphire laser. The pulses were delivered at frequency $11 \mathrm{kHz}$ to match the maximum repetition rate of the detection apparatus. The polarization of pump beam was adjusted by means of a half-wave plate. The collection of non-collinear frequency-degenerated (at $532 \mathrm{~nm}$ ) signal and idler fields was performed $20 \mathrm{~cm}$ beyond the nonlinear crystal using bandpass filters followed by multi-mode fibers $(600-\mu \mathrm{m}$-core diameter) and two HPDs (mod. R10467U-40, Hamamatsu, Japan)13,20. The output of each HPD was amplified, synchronously integrated and digitized. The experimental results confirmed a convex dependence of covariance $c_{m, \delta v}$ on widths $\delta v_{s}$ and $\delta v_{i}$. This dependence is demonstrated in Fig. 2(a) for typical experimental data giving $\left\langle v_{s}\right\rangle_{a}=0.534,\left\langle v_{i}\right\rangle_{a}=0.545$, $\left\langle\left(\Delta v_{s}\right)^{2}\right\rangle_{a}=0.557,\left\langle\left(\Delta v_{i}\right)^{2}\right\rangle_{a}=0.572$, and $\left\langle\Delta v_{s} \Delta v_{i}\right\rangle_{a}=$ 0.053 . If values of QDE's $\eta_{s}$ and $\eta_{i}$ are close to each other and we measure a TB with small amount of noise photons, the mean values of measured voltages depend
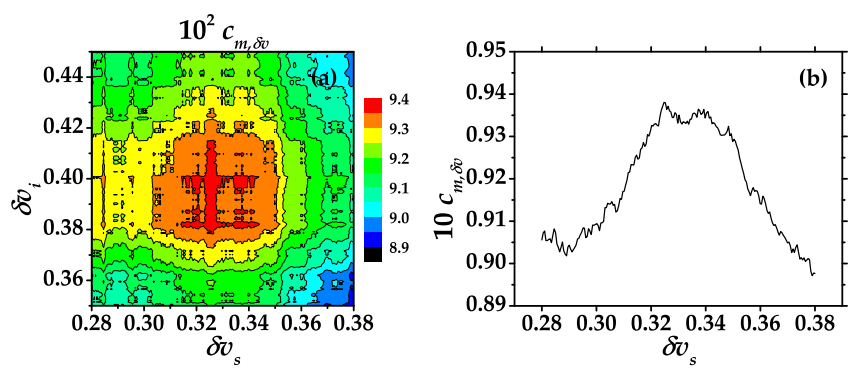

FIG. 2. (a) Topo graph of covariance $c_{m, \delta v}$ as a function of windows' widths $\delta v_{s}$ and $\delta v_{i}$. (b) Covariance $c_{m, \delta v}$ depending on $\delta v_{s}$ assuming $\delta v_{i}=r \delta v_{s}, r=1.17$. In both cases, the maximum of $c_{m, \delta v}\left(9.37 \times 10^{-2}\right)$ is reached for $\delta v_{s}=0.326$ and $\delta v_{i}=0.375$.

linearly on detector amplification and so also on windows' widths. We can thus assume that

$$
\delta v_{i} / \delta v_{s} \approx\left\langle v_{i}\right\rangle_{a} /\left\langle v_{s}\right\rangle_{a} \equiv r .
$$

This further simplifies the analysis and improves precision in the determination of windows' widths $\delta v_{s}$ and $\delta v_{i}$ [see Fig. 2(b)].

Once the windows' widths $\delta v_{s}$ and $\delta v_{i}$ are fixed, the QDEs $\eta_{s}$ and $\eta_{i}$ together with the joint signal-idler photon-number distribution $p\left(n_{s}, n_{i}\right)$ [PND] describing the generated TB in front of the detectors can be determined using the experimental photo-electron histogram $f\left(m_{s}, m_{i}\right)$. Following the approach described in ${ }^{18}$, TBs are considered as composed of three independent components characterizing ideal paired field (index $p$ ), signal noise photon field $(s)$ and idler noise photon field (i). The components are assumed in multi-mode thermal states given for the photon-paired field by the fundamental theory ${ }^{21.22}$. As such they are composed of $M_{c}$ independent equally-populated modes with $b_{c}$ mean photon (-pair) numbers per mode $(c=p, s, i)$. We note that if the number $M$ of modes is sufficiently large (usually $>5$ ), a field spectral profile composed of differently populated modes plays only a negligible role in the determination of $\mathrm{PND}^{14,23}$. The corresponding $\mathrm{PND} p\left(n_{s}, n_{i}\right)$ can be written as a two-fold convolution comprising three Mandel-Rice distributions 14,18 :

$$
\begin{aligned}
p\left(n_{s}, n_{i}\right)= & \sum_{n=0}^{\min \left[n_{s}, n_{i}\right]} p\left(n_{s}-n ; M_{s}, b_{s}\right) \\
& \times p\left(n_{i}-n ; M_{i}, b_{i}\right) p\left(n ; M_{p}, b_{p}\right),
\end{aligned}
$$

where $p(n ; M, b)=\Gamma(n+M) /[n ! \Gamma(M)] b^{n} /(1+b)^{n+M} ; \Gamma$ is the $\Gamma$-function. Numbers $M_{c}$ of modes and mean photon (-pair) numbers per mode $b_{c}$ can be derived from the first and second photon (-pair) moments of these components using the expressions for thermal fields.

$$
M_{c}=\frac{\left\langle n_{c}\right\rangle^{2}}{\left\langle\left(\Delta n_{c}\right)^{2}\right\rangle-\left\langle n_{c}\right\rangle}, \quad b_{c}=\frac{\left\langle\left(\Delta n_{c}\right)^{2}\right\rangle}{\left\langle n_{c}\right\rangle}-1 .
$$

Photon (-pair) moments written in Eq. (6) and experimental photo-electron moments in Eq. (3) fulfil the 
relations established by photo-detection theory ${ }^{14}$. In this theory, QDEs $\eta_{s}$ and $\eta_{i}$ are introduced as detector parameters and the needed relations are derived as $(c=s, i)$ :

$$
\begin{gathered}
\eta_{c}\left[\left\langle n_{p}\right\rangle+\left\langle n_{c}\right\rangle\right]=\left\langle m_{c}\right\rangle, \quad \eta_{s} \eta_{i}\left\langle\left(\Delta n_{p}\right)^{2}\right\rangle=\left\langle\Delta m_{s} \Delta m_{i}\right\rangle \\
\eta_{c}^{2}\left[\left\langle\left(\Delta n_{p}\right)^{2}\right\rangle+\left\langle\left(\Delta n_{c}\right)^{2}\right\rangle+\frac{1-\eta_{c}}{\eta_{c}}\left(\left\langle n_{p}\right\rangle+\left\langle n_{c}\right\rangle\right)\right] \\
=\left\langle\left(\Delta m_{c}\right)^{2}\right\rangle .
\end{gathered}
$$

In five Eqs. (7), there occur eight unknown parameters: six parameters of TBs and two QDEs. We express the photon moments in the terms of photo-electron moments and mean photon-pair number $\langle n\rangle_{p}$, which is taken as an independent parameter $(c=s, i)$ :

$$
\begin{array}{r}
\left\langle n_{c}\right\rangle=\frac{\left\langle m_{c}\right\rangle}{\eta_{c}}-\left\langle n_{p}\right\rangle,\left\langle\left(\Delta n_{p}\right)^{2}\right\rangle=\frac{\left\langle\Delta m_{s} \Delta m_{i}\right\rangle}{\eta_{s} \eta_{i}}, \\
\left\langle\left(\Delta n_{c}\right)^{2}\right\rangle=\frac{\left\langle\left(\Delta m_{c}\right)^{2}\right\rangle}{\eta_{c}^{2}}-\frac{\left\langle\Delta m_{s} \Delta m_{i}\right\rangle}{\eta_{s} \eta_{i}}-\frac{1-\eta_{c}}{\eta_{c}^{2}}\left\langle m_{c}\right\rangle .(8)
\end{array}
$$

Values of the remaining three unknown parameters, $\langle n\rangle_{p}$, $\eta_{s}$ and $\eta_{i}$, can be found by minimizing the declinations between the theoretical and experimental photo-electron histograms $p_{m}$ and $f$ quantified by function $D$ :

$$
D=\sqrt{\sum_{m_{s}, m_{i}=0}^{\infty}\left[p_{m}\left(m_{s}, m_{i}\right)-f\left(m_{s}, m_{i}\right)\right]^{2}} .
$$

The theoretical photo-electron histogram $p_{m}$ can be obtained from the PND $p$ in Eq. (6) provided that HPDs are described by the Bernoulli distribution $T_{k} 24$ :

$$
\begin{aligned}
& p_{c}\left(m_{s}, m_{i}\right)=\sum_{n_{s}, n_{i}=0}^{\infty} T_{s}\left(m_{s}, n_{s}\right) T_{i}\left(m_{i}, n_{i}\right) p\left(n_{s}, n_{i}\right) \\
& T_{k}(m, n)=\left(\begin{array}{c}
m \\
n
\end{array}\right) \eta_{k}^{m}\left(1-\eta_{k}\right)^{m-n}, \quad k=s, i
\end{aligned}
$$

The declination function $D$ in Eq. (9) depends on $\langle n\rangle_{p}$, $\eta_{s}$ and $\eta_{i}$ and attains its minimum that identifies suitable values for the unknown parameters. This is documented in Fig. 3(a) for the experimental data analyzed in Fig. 2 assuming windows' widths $\delta v_{s}$ and $\delta v_{i}$ maximizing the covariance $c_{m, \delta v}$. According to the graph in Fig. 31(a), the minimum of $D$ is reached for $\eta_{s}=0.085 \pm 0.002$ and $\eta_{i}=0.086 \pm 0.002$. We note that QDEs inevitably incorporate also collection efficiencies as the TB in front of the detectors (and not beyond the crystal) is considered. The reconstructed TB is characterized by the following values of its parameters determined by Eqs. (8) and (6): $M_{p}=38, b_{p}=0.16, M_{s}=1.4 \times 10^{-3}, b_{s}=39$, $M_{i}=5.0 \times 10^{-3}$ and $b_{i}=24$. Its joint signal-idler PND $p\left(n_{s}, n_{i}\right)$ is shown in Fig. $3(\mathrm{~b})$. On average, the TB is composed of 6.2 photon pairs distributed over 38 modes, 0.05 signal noise photons and 0.11 idler noise photons. This means that less than $2 \%$ of photons in the beam are noise photons. The numbers $M_{s}$ and $M_{i}$ of signal and idler noise modes given by the fitting method are

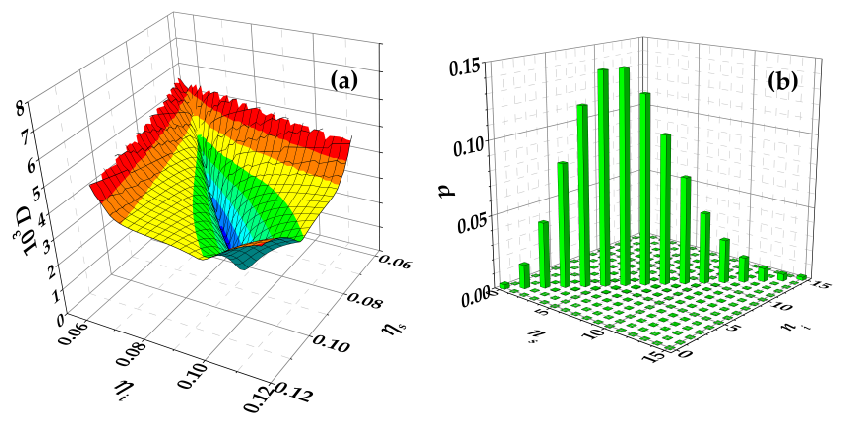

FIG. 3. (a) Minimum of declination function $D$ determined over the allowed values of $\left\langle n_{p}\right\rangle$ in dependence on QDEs $\eta_{s}$ and $\eta_{i}$. In areas close to the $\eta_{s}$ and $\eta_{i}$ axes, Eqs. (7) have no solution. In (b), signal-idler PND $p\left(n_{s}, n_{i}\right)$ for optimal values of QDEs $\eta_{s}=0.085$ and $\eta_{i}=0.086$ is shown.

less than 1. This means that the corresponding superGaussian PNDs have high probabilities at the zero photon number and low but long tails. We attribute this form of PNDs to distortions of weak electronic signals inside the detection chain and not exactly set zeroes at the voltages axes. This means that the originally assumed noise optical fields considered as noise photo-electron signals contain an important contribution from electronic noise that even changes the type of their statistics. Covariance between the signal and idler photon numbers is $0.76 \pm 0.02$. Noise reduction factor $R$ quantifying subshot-noise correlations between the signal and idler photon numbers ${ }^{18}$ equals $0.37 \pm 0.02$ indicating a larger role of noise photons in the TB.

The presented two-step analysis can be simplified provided that the noise signal and idler fields are weak compared to that of photon pairs and can be omitted. In this case, both steps can be combined together giving the following formulas for the theoretical $\left[\left\langle n_{p}\right\rangle,\left\langle\left(\Delta n_{p}\right)^{2}\right\rangle\right]$ and experimental $\left[\left\langle v_{s}\right\rangle_{a},\left\langle v_{i}\right\rangle_{a},\left\langle\left(\Delta v_{s}\right)^{2}\right\rangle_{a},\left\langle\left(\Delta v_{i}\right)^{2}\right\rangle_{a}\right.$, $\left.\left\langle\Delta v_{s} \Delta v_{i}\right\rangle_{a}\right]$ moments $(c=s, i)$ :

$$
\begin{gathered}
\eta_{c} \delta v_{c}\left\langle n_{p}\right\rangle=\left\langle v_{c}\right\rangle_{a}, \quad \eta_{s} \delta v_{s} \eta_{i} \delta v_{i}\left\langle\left(\Delta n_{p}\right)^{2}\right\rangle=\left\langle\Delta v_{s} \Delta v_{i}\right\rangle_{a} \\
\eta_{c}^{2} \delta v_{c}^{2}\left[\left\langle\left(\Delta n_{p}\right)^{2}\right\rangle+\frac{1-\eta_{c}}{\eta_{c}}\left\langle n_{p}\right\rangle\right]=\left\langle\left(\Delta v_{c}\right)^{2}\right\rangle_{a}
\end{gathered}
$$

Equations (12) represent five constrains for allowed values of six unknown parameters. Two of them characterize the paired field $\left[\left\langle n_{p}\right\rangle,\left\langle\left(\Delta n_{p}\right)^{2}\right\rangle\right]$ whereas the remaining four parameters describe the detection process. Relations in Eqs. (12) can be rewritten such that the unknown parameters are expressed as functions of QDE $\eta_{s}$. An optimal value of QDE $\eta_{s}$ is chosen, similarly as in the general approach, such that the declination function $D$ in Eq. (9) minimizes.

Equations (12) are rearranged as follows. First, the ratio of equations for $\left\langle v_{s}\right\rangle$ and $\left\langle v_{i}\right\rangle$ provides the relation:

$$
\eta_{i} \delta v_{i}=r \eta_{s} \delta v_{s}
$$

where $r$ is defined in Eq. (4). By using $r$, the equations 
for second moments in (12) can be recast as:

$$
\begin{aligned}
\eta_{s}^{2} \delta v_{s}^{2}\left[\left\langle\left(\Delta n_{p}\right)^{2}\right\rangle+\frac{1-\eta_{s}}{\delta v_{s} \eta_{s}^{2}}\left\langle v_{s}\right\rangle_{a}\right] & =\left\langle\left(\Delta v_{s}\right)^{2}\right\rangle_{a}, \\
\eta_{s}^{2} \delta v_{s}^{2}\left[\left\langle\left(\Delta n_{p}\right)^{2}\right\rangle+\frac{1-\eta_{i}}{\delta v_{s} \eta_{s} \eta_{i}}\left\langle v_{s}\right\rangle_{a}\right] & =\frac{\left\langle\left(\Delta v_{i}\right)^{2}\right\rangle_{a}}{r^{2}} \\
& \equiv\left\langle\left(\Delta v_{i}\right)^{2}\right\rangle_{r}, \\
\eta_{s}^{2} \delta v_{s}^{2}\left\langle\left(\Delta n_{p}\right)^{2}\right\rangle & =\frac{\left\langle\Delta v_{s} \Delta v_{i}\right\rangle_{a}}{r} \\
& \equiv\left\langle\Delta v_{s} \Delta v_{i}\right\rangle_{r}
\end{aligned}
$$

Combining the first and third equations in (14) the expression for $\delta v_{s}$ as a function of QDE $\eta_{s}$ can be reached:

$$
\delta v_{s}=\frac{\left\langle\left(\Delta v_{s}\right)^{2}\right\rangle_{a}-\left\langle\Delta v_{s} \Delta v_{i}\right\rangle_{r}}{\left(1-\eta_{s}\right)\left\langle v_{s}\right\rangle_{a}}
$$

Also the relation for QDE $\eta_{i}$ can be recovered:

$$
\eta_{i}=\frac{\left[\left\langle\left(\Delta v_{s}\right)^{2}\right\rangle_{a}-\left\langle\Delta v_{s} \Delta v_{i}\right\rangle_{r}\right] \eta_{s}}{\left\langle\left(\Delta v_{i}\right)^{2}\right\rangle_{r}-\left\langle\Delta v_{s} \Delta v_{i}\right\rangle_{r}+\eta_{s}\left[\left\langle\left(\Delta v_{s}\right)^{2}\right\rangle_{a}-\left\langle\left(\Delta v_{i}\right)^{2}\right\rangle_{r}\right]} .
$$

The voltage window's width $\delta v_{i}$ is then obtained as:

$$
\delta v_{i}=r \delta v_{s} \eta_{s} / \eta_{i}
$$

The photon-number moments of the paired field can be easily determined using Eqs. (12):

$$
\left\langle n_{p}\right\rangle=\frac{\left\langle v_{s}\right\rangle_{a}}{\eta_{s} \delta v_{s}}, \quad\left\langle\left(\Delta n_{p}\right)^{2}\right\rangle=\frac{\left\langle\Delta v_{s} \Delta v_{i}\right\rangle_{a}}{\eta_{s} \delta v_{s} \eta_{i} \delta v_{i}}
$$

Finally, the number $M_{p}$ of modes and their average mean photon-pair number $b_{p}$ are obtained by using Eqs. (6).

The analysis of the data leading to Fig. 2 shows that the declination function $D$ decreases with QDE $\eta_{s}$ in the interval of allowed values $\eta_{s}$. Also the remaining three parameters, $\eta_{i}, \delta v_{s}$ and $\delta v_{i}$, are monotonous functions of the QDE $\eta_{s}$ which makes the optimization procedure stable. The declination function $D$ thus attains its minimum value at the border, where $\eta_{s}=0.09 \pm 0.005$, $\eta_{i}=0.086 \pm 0.005, \delta v_{s}=0.341$, and $\delta v_{i}=0.422$. The TB contains on average 6.1 photon pairs found in $M_{p}=1800$ independent modes $\left(b_{p}=3.3 \times 10^{-3}\right)$. Comparison of these results with the previous ones reveals that the simplified method is able to determine QDEs with the relative precision better than $5 \%$ for the TB containing $2 \%$ of noise photons. We estimate that relative precision better than $10 \%$ is reached provided that the noise photons form less than $4 \%$ of a TB. The method also provides reasonable mean photon-pair numbers $\left\langle n_{p}\right\rangle$. On the other hand, it overestimates windows' widths $\delta v_{s}$ and $\delta v_{i}$ and assigns larger numbers $M_{p}$ of independent photon-pair modes. This originates in the presence of non-negligible amount of noise photons in the analyzed TB. However, these drawbacks are acceptable and they are outweighed by relative simplicity of the simplified method compared to the general two-step approach. The simplified method is also more suitable for the analysis of data collected with more intense TBs.

In conclusion, we have developed and experimentally verified a method allowing absolute detector calibration of photon-number-resolving detectors with analog output using twin beams. It gives detection efficiencies with high precision provided that the noise is sufficiently small. Especially electronic noise is not treated by the method in its full complexity and its more rigorous description would give more insight into measurement uncertainties and result in the improved precision. The method also reveals parameters of twin beams. A simplified approach suitable for more intense twin beams has been suggested. Experimental conditions for its use have been found.

Support by projects P205/12/0382 of GA ČR, CZ.1.05/2.1.00/03.0058 and CZ.1.07/2.3.00/20.0058 of MŠMT ČR and MIUR (FIRB LiCHIS - RBFR10YQ3H) are acknowledged.

${ }^{1}$ L. Mandel and E. Wolf, Optical Coherence and Quantum Optics (Cambridge Univ. Press, Cambridge, 1995).

${ }^{2}$ O. Haderka, M. Hamar, and J. Peřina Jr., Eur. Phys. J. D 28, 149 (2004).

${ }^{3}$ J. Řeháček, Z. Hradil, O. Haderka, J. Peřina Jr., and M. Hamar, Phys. Rev. A 67, 061801(R) (2003).

${ }^{4}$ M. J. Fitch, B. C. Jacobs, T. B. Pittman, and J. D. Franson, Phys. Rev. A 68, 043814 (2003).

${ }^{5}$ O. Haderka, J. Peřina Jr., M. Hamar, and J. Peřina, Phys. Rev. A 71, 033815 (2005).

${ }^{6}$ A. J. Miller, S. W. Nam, J. M. Martinis, and A. V. Sergienko, Appl. Phys. Lett. 83, 791 (2003).

${ }^{7}$ D. Fukuda, G. Fujii, T. Numata, K. Amemiya, A. Yoshizawa, H. Tsuchida, H. Fujino, H. Ishii, T. Itatani, S. Inoue, et al., Opt. Express 19, 870 (2011).

${ }^{8}$ A. Avella, G. Brida, I. P. Degiovanni, M. Genovese, M. Gramegna, L. Lolli, E. Monticone, C. Portesi, M. Rajteri, M. L. Rastello, et al., Opt. Express 19, 23249 (2011).

${ }^{9}$ L. A. Jiang, E. A. Dauler, and J. T. Chang, Phys. Rev. A 75, 062325 (2007).

${ }^{10}$ I. Afek, A. Natan, O. Ambar, and Y. Silberberg, Phys. Rev. A 79, 043830 (2009).

${ }^{11}$ M. Ramilli, A. Allevi, V. Chmill, M. Bondani, M. Caccia, and A. Andreoni, J. Opt. Soc. Am. B 27, 852 (2010).

${ }^{12}$ A. Allevi, M. Bondani, and A. Andreoni, Opt. Lett. 35, 1707 (2010).

${ }^{13}$ M. Bondani, A. Allevi, A. Agliati, and A. Andreoni, J. Mod. Opt. 56, 226 (2009).

${ }^{14}$ J. Peřina, Quantum Statistics of Linear and Nonlinear Optical Phenomena (Kluwer, Dordrecht, 1991).

${ }^{15}$ A. A. Malygin, A. N. Penin, and A. V. Sergienko, Pisma Zh. Eksp. Teor. Fiz. 33, 493 (1981).

${ }^{16}$ A. Migdall, Physics Today 52, 41 (1999).

${ }^{17}$ G. Brida, I. P. Degiovanni, M. Genovese, M. L. Rastello, and I. R. Berchera, Opt. Express 18, 20572 (2010).

${ }^{18}$ J. Peřina Jr., O. Haderka, M. Hamar, and V. Michálek, Opt. Lett. 37, 2475 (2012).

${ }^{19}$ J. Peřina Jr., O. Haderka, V. Michálek, and M. Hamar, Phys. Rev. A 87, 022108 (2013).

${ }^{20}$ A. Andreoni and M. Bondani, Phys. Rev. A 80, 013819 (2009).

${ }^{21}$ J. Peřina and J. Křepelka, J. Opt. B: Quant. Semiclass. Opt. 7, 246 (2005).

${ }^{22}$ J. Peřina and J. Křepelka, Opt. Commun. 265, 632 (2006).

${ }^{23}$ E. A. Goldschmidt, F. Piacentini, I. Ruo-Berchera, S. V. Polyakov, S. Peters, S. Kück, G. Brida, I. P. Degiovanni, A. Migdall, and M. Genovese, Phys. Rev. A 88, 016822 (2013).

${ }^{24}$ J. Peřina Jr., M. Hamar, V. Michálek, and O. Haderka, Phys. Rev. A 85, 023816 (2012). 